\title{
First GPS-TEC evidence for the wave structure excited by the solar terminator
}

\author{
E. L. Afraimovich \\ Institute of Solar-Terrestrial Physics SB PAS, P.O. 291, Irkutsk, 664033, Russia
}

(Received March 25, 2008; Revised May 12, 2008; Accepted May 14, 2008; Online published September 8, 2008)

\begin{abstract}
Using TEC measurements from the global network of GPS receivers, we have obtained the first evidence for the wave structure excited by the solar terminator (ST), moving over the USA, Europe, and Japan. Two main types of the observed TEC disturbance were found: large-scale (LS) 60-min variations with an amplitude of about 0.5-1 TECU and medium-scale (MS) 15-min variations with an amplitude of about 0.05-0.1 TECU. The first type of disturbances was predicted in theoretical investigations and registered earlier using different methods of ionosphere radio sounding. The second type of the observed TEC disturbance is wave packets (WPs) generated when the time derivative of TEC is at its maximum. These WPs have duration of about $1-2 \mathrm{~h}$ and a time shift of about 1.5-2.5 h after the ST appearance at an altitude of $100 \mathrm{~km}$. That ST-generated wave packets have been found for the first time. LS TEC disturbances and MS WPs are space-fixed along the ST line over a distance exceeding $1600 \mathrm{~km}$.
\end{abstract}

Key words: Solar terminator, total electron content, wave packets, GPS.

\section{Introduction}

Recent investigations have shown that movement of the solar terminator (ST) causes the generation of acousticgravity waves (STAGW), turbulence, and instabilities in the ionosphere plasma (Beer, 1978; Cot and Teitelbaum, 1980; Somsikov, 1983, 1987; Somsikov and Ganguly, 1995; Dungenbaeva and Ganguly, 2004; Antonova et al., 2006). It is worth noting that among all of the sources of gravity waves, the moving ST has a special status, since it is a predictable phenomenon, whose characteristics are well known. If the $\mathrm{ST}$ is considered to be as a stable and repetitive source of AGWs, one can derive information about atmospheric conditions from the response of the medium to this input.

The great variety of ST-linked phenomena in the atmosphere has resulted in a number of studies being carried out on the analysis of ionosphere parameter variations obtained by different methods of ionosphere sounding (Bezrodny and Yampolski, 1976; Popov and Yampolski, 1981; Galushko and Yampolski, 1983; Mishin et al., 1991; Drobzhev et al., 1992; Bezotosnyi et al., 1994; Beley et al., 1995; Sobral et al., 1997; Galushko et al., 1998; Hocke and Igarashi, 2002; Galushko et al., 2003). However, virtually all experimental data were obtained using indirect methods for analyzing the spectrum of ionosphere parameter variations, which can result from a number of factors. This has caused difficulties in the reliable identification of STAGWs because, in general, AGWs can be generated by different sources of either natural or of anthropogenic origin (Hocke and Schlegel, 1996).

To identify ST-generated wave disturbances it is insufficient to register the time dependence of ionosphere parameters or their spectrum. Rather, it is necessary to measure

Copyright (c) The Society of Geomagnetism and Earth, Planetary and Space Sciences (SGEPSS); The Seismological Society of Japan; The Volcanological Society of Japan; The Geodetic Society of Japan; The Japanese Society for Planetary Sciences; TERRAPUB the spatial structure of these disturbances and to compare it with spatial-temporal characteristics of ST. Another important requirement implies the continuous, global character of the observations.

Considerable progress has recently been achieved in the study of ionosphere irregularities using the new technology of GPS radio sounding, which allows data to be obtained on variations of the total electron content (TEC) with high spatial and temporal resolution. The ISTP SB RAS developed methods and a technology for the global GPS detector of ionosphere disturbances, whose high sensitivity enables the analysis of ionosphere disturbances with amplitudes as high as $10^{-3}$ of the TEC background value (Afraimovich and Perevalova, 2006). Compared to classical radio probing tools for the ionosphere, this technology is the first to ensure real continuous and global coverage providing monitoring of ionosphere disturbances.

The goal of this paper is to present the first evidence for the TEC wave structure excited by the solar terminator. The data were obtained at the global network of two-frequency GPS receivers. As examples we have chosen day 291 (October 18, 2001) for the USA and Europe, and several days for Japan-that is, regions with dense GPS networks.

\section{GPS Data Processing and Results}

The data we used in our work are available in the standard RINEX format with sampling intervals of $30 \mathrm{~s}$ from sites (http://sopac.ucsd.edu/cgi-bin/dbDataByDate.cgi) and (ftp://terras.gsi.go.jp/data/GPS_products/). Standard GPS technology provides the means for detecting wave disturbances based on phase measurements of slant TEC $I_{\mathrm{s}}$. Methods for calculating $I_{\mathrm{s}}$ using GPS phase measurements are described in detail in Hofmann-Wellenhof et al. (1992), Afraimovich et al. (2003), and Afraimovich and Perevalova (2006). We reproduce here only the final formula for phase 
measurements:

$$
I_{\mathrm{s}}=\frac{1}{40.308} \frac{f_{1}^{2} f_{2}^{2}}{f_{1}^{2}-f_{2}^{2}}\left[\left(L_{1} \lambda_{1}-L_{2} \lambda_{2}\right)+\text { const }+n L\right]
$$

where $L_{1} \lambda_{1}$ and $L_{2} \lambda_{2}$ are additional paths of the radio signal caused by the phase delay in the ionosphere $(\mathrm{m}) ; L_{1}$ and $L_{2}$ are the number of phase rotations at the frequencies $f_{1}$ and $f_{2} ; \lambda_{1}$ and $\lambda_{2}$ stand for the corresponding wavelengths, (m); const is the unknown initial phase ambiguity, $(\mathrm{m}) ; n L$ is error in determining the phase path, $(\mathrm{m})$.

Phase measurements in the GPS can be made with a high degree of accuracy corresponding to the error of TEC determination of at least $10^{14} \mathrm{~m}^{-2}$ when averaged on a 30-s time interval, although with some uncertainty of the initial value of TEC (Hofmann-Wellenhof et al., 1992). This makes it possible to detect ionization irregularities and wave processes in the ionosphere over a wide range of amplitudes (up to $10^{-4}$ of the diurnal TEC variation and periods (from $24 \mathrm{~h}$ to $5 \mathrm{~min}$ ). The unit of TEC, which is equal to $10^{16} \mathrm{~m}^{-2}$ (TECU) and widely accepted in the literature, will be used in the following.

To normalize the amplitude of TEC disturbances, we use the transformation of slant TEC into the equivalent vertical value $I(t)$ (Klobuchar, 1986):

$$
I(t)=I_{\mathrm{s}}(t) \times \cos \left[\arcsin \left(\frac{r_{\mathrm{z}}}{r_{\mathrm{z}}+h_{\max }} \cos \theta_{\mathrm{s}}\right)\right]
$$

where $r_{\mathrm{z}}$ is the Earth's radius, and $h_{\max }=300$ or $400 \mathrm{~km}$ is the assumed altitude of the ionospheric $F_{2}$ layer maximum. To obtain the absolute vertical TEC, we use the CODG global ionospheric map GIM in the IONEX format (ftp://cddisa.gsfc.nasa.gov/pub/gps/products/ionex/).

To eliminate the variations of the regular ionosphere and trends caused by the satellite travel, we first smooth the initial series with the selected time window of 2 min and remove their linear trend with a window of about $90 \mathrm{~min}$. We thus obtain the TEC variations $d I(t)$ in the range of periods 2-90 min corresponding to the AGW range of periods (Hocke and Schlegel, 1996).

Let us consider the first example. The geomagnetic situation on 18 October 2001 can be characterized as quiet: the $K_{\mathrm{p}}$ index varied from 1.0 to 1.7 (http://www.ukssdc.ac.uk/wdcc1/wdc_menu.html). The geometry of GPS measurements during morning ST moving over the U.S. West is shown in Fig. 1(a). The chosen GPS sites are marked by triangles; the names of the sites are written nearby. The coordinates of the sites are not given for reasons of space. Solid lines show the trajectory of subionospheric points for the chosen GPS satellite PRN-14 at $h=400 \mathrm{~km}$; the motion direction is marked by arrays. The thick dotted gray line and arrow show the ST position at $H=100 \mathrm{~km}$ at $13.2 \mathrm{UT}$ and direction of the ST motion. We approximately choose the altitude $H=100 \mathrm{~km}$ for the ST location, synchronization of ST appearance, and TEC disturbance commencements. Hence, the GPS ray at the chosen PRN-14 intersects the ST front.

For the PRN-14 and CTMS, MONB, KELS sites, Fig. 1(b, d) presents time dependencies of the initial TEC series $I(t)$ smoothed with the time window of $2 \mathrm{~min}$ and

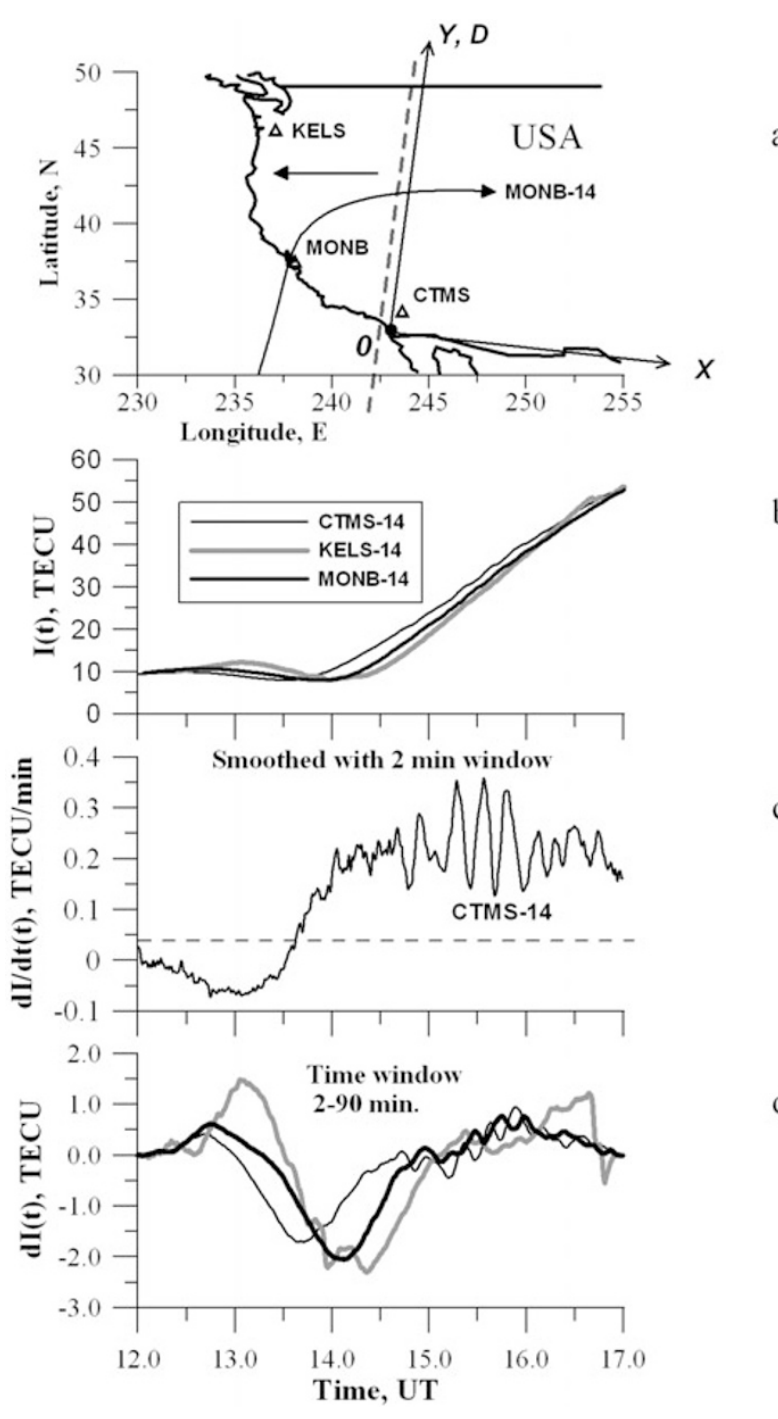

a.

b.

Fig. 1. Experimental geometry of GPS measurements during morning ST over the U.S. West on October 18, 2001 (a); Time dependencies of the initial TEC series $I(t)(b)$; Time derivative of the initial TEC series $d I(t) / d t(\mathrm{c})$; TEC series $d I(t)$ filtered in the range of 2-90 min (d).

TEC series $d I(t)$ filtered in the range of 2-90 min, respectively. Figure 1(d) presents two main types of the observed TEC disturbance: long-period (of about 60-min) variations with an amplitude of about $0.5-1$ TECU and short-period (of about 15-min) variation with an amplitude of about 0.05-0.1 TECU. Long-period TEC variations are similar in shape, but they are shifted in time relative to each other according to ST appearance at different points.

Figure 1(c) presents the time derivative of the initial TEC series $\frac{d I(t)}{d t}$ smoothed with the time window of $2 \mathrm{~min}$. Shortperiod oscillations of the TEC time derivative have the form of the wave train or wave packets (WPs). The WPs arise when the time derivative of the initial TEC series $\frac{d I(t)}{d t}$ amount to its maximum.

Figure 2(b) shows the LS structure of TEC disturbances along the morning ST over the western USA with the dense network of GPS sites. The initial TEC series $I(t)$ for PRN14 (Fig. 2(b)) and TEC variations $d I(t)$ filtered within 20 $90 \mathrm{~min}$ (Fig. 2(a)) are presented at different distances $D$ from the center $O\left(33^{\circ} \mathrm{N} ; 243^{\circ} \mathrm{E}\right)$ of the topocentric coordi- 


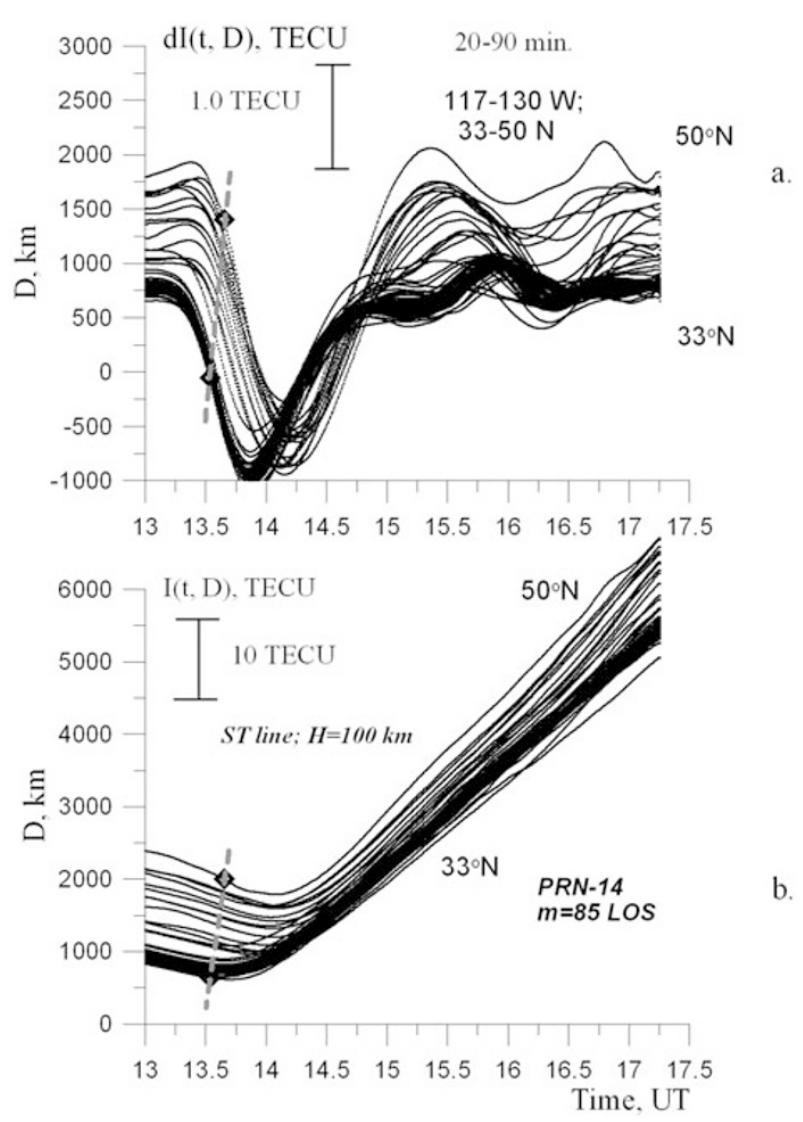

Fig. 2. Large scale structure of TEC disturbances along morning ST. Time dependencies of the initial TEC series $I(t)(\mathrm{b})$; TEC series $d I(t)$ filtered in the range of 20-90 $\mathrm{min}$ (a).

nate system $(X O Y)$, with coordinate axes $(X, Y)$ directed perpendicular to and along the ST respectively (Fig. 1(a)). Thick gray dotted lines indicate the morning ST line at $H=100 \mathrm{~km}$.

The distance $D$ positive value corresponds to the axis $Y$ one. Figure 2 presents the initial and filtered TEC as

$$
D_{i}(t)=D_{0 i}+k_{1} I(t) ; \quad d D_{i}(t)=D_{0 i}+k_{2} d I(t)
$$

where $D_{0, i}$ is the coordinate $Y$ value for each GPS site located along the ST line (axis $Y$ ); $i$ is the number of GPS sites (or line of sight, LOS, for the chosen satellite PRN14); $i=1,2 \ldots N=85 ; k_{1}$ and $k_{2}$ are aspect ratios for $I(t)$ and $d I(t)$, respectively (marked by vertical bars in Fig. 2).

Figure 2(a,b) show that the observed LS disturbances are space-fixed along the ST line over the distance $D$ exceeding $1600 \mathrm{~km}$ and travel with ST.

Figure 3 presents the MS structure for different GPS sites located along ST line (the U.S. West-left, U.S. East-middle, Europe-right) on 18 October 2001. The TEC oscillations $d I(t)$ are filtered from the initial TEC series in the time period of 2-20 min using the eight-order Butterworth band-pass filter. The diamonds mark the instant of the ST appearance at $H=100 \mathrm{~km}$. The averaged diurnal dependences of the vertical TEC for the center of the said regions reconstructed from CODG IONEX data (ftp://cddisa.gsfc.nasa.gov/pub/gps/products/ionex/) are plotted in the panels ( (a): the horizontal gray lines on the time axis indicate the time interval using in panels $(b-i))$.
Names and coordinates of GPS sites and observed PRN are given on each panel. As is seen from Fig. 3, the observed MS TEC pulsations are wave packets with a duration of about 1-2 h. These WPs appeared about 1.5-2.5 h later than the ST at $H=100 \mathrm{~km}$. The WPs arise on the stage of TEC fast increasing for morning hours (Fig. 3(a)), which corresponds to the maximum of the time derivative of the initial TEC series $\frac{d I(t)}{d t}$ for chosen PRN (Fig. 1(b)). The absolute amplitude of TEC disturbances (of about 0.05-0.1 TECU) is in accord with the relative amplitude $\frac{d I}{I}$ of about $0.2 \%$. The main period of WPs varies from 12 to 17 min. Similar to the LS structure, WPs are space-fixed along the ST line over a distance exceeding $1000 \mathrm{~km}$.

We choose the geomagnetic quiet day of October 18, $2001\left(K_{\mathrm{p}}=1-1.7\right)$ for our detailed analysis. However, we obtained similar results for moderate geomagnetic disturbed days as well (Fig. 4). One can see TEC WPs over Japan for the disturbed days on 5 September 2004-left; on 25 September 2003-middle; on 15 November 2006-right. The well-known major earthquakes occurred in these days (http://earthquake.usgs.gov/regional/world/historical.php). The black triangles in Fig. 4 mark the main shocks. However, the WPs did not result from the earthquakes; rather they were generated by the ST. Figures 3 and 4 confirm that the ST-generated WPs were detected in different levels of geomagnetic activity and over different regions of the Earth.

\section{Discussion and Conclusion}

These data are the first evidence for the wave structure excited by the ST using TEC measurements from the global network of GPS receivers. Two main types of observed TEC disturbance were found: LS variations with an amplitude of about 0.5-1 TECU and MS wave packets with an amplitude of about 0.05-0.1 TECU. These structures are space-fixed along the ST line over a distance exceeding $1600 \mathrm{~km}$. Hence, these observations confirm that the ST is a stable and repetitive source of ionospheric wave disturbances and also show that the GPS TEC representation of the ST-generated ionospheric structure suggests better possibilities for investigating this phenomenon.

This analysis revealed that the relative amplitude $\frac{d I}{I}$ of the variation in ST-generated LS disturbances in the middle latitudes can be compared with the TEC variation amplitude increase during a magnetic storm main phase and corresponds to changes in the local electron density up to 5-7\% (Afraimovich et al., 2008). The obtained results concerning the LS structure agree with theoretical indications of ST effects (Cot and Teitelbaum, 1980; Somsikov, 1983, 1987; Somsikov and Ganguly, 1995; Dungenbaeva and Ganguly, 2004; Antonova et al., 2006) and do not contradict the experimental results obtained by Mishin et al. (1991), Beley et al. (1995), and Galushko et al. (1998, 2003). In particular, these results confirm the conclusion by Galushko et al. (1998) that ST-generated AGWs can be observed at sunrise better than at sunset (corresponding data will be presented in future publications). Using the GPS/MET observation at heights from 300 to $600 \mathrm{~km}$, Hocke and Igarashi (2002) found the LS structure that may result from the disturbance 


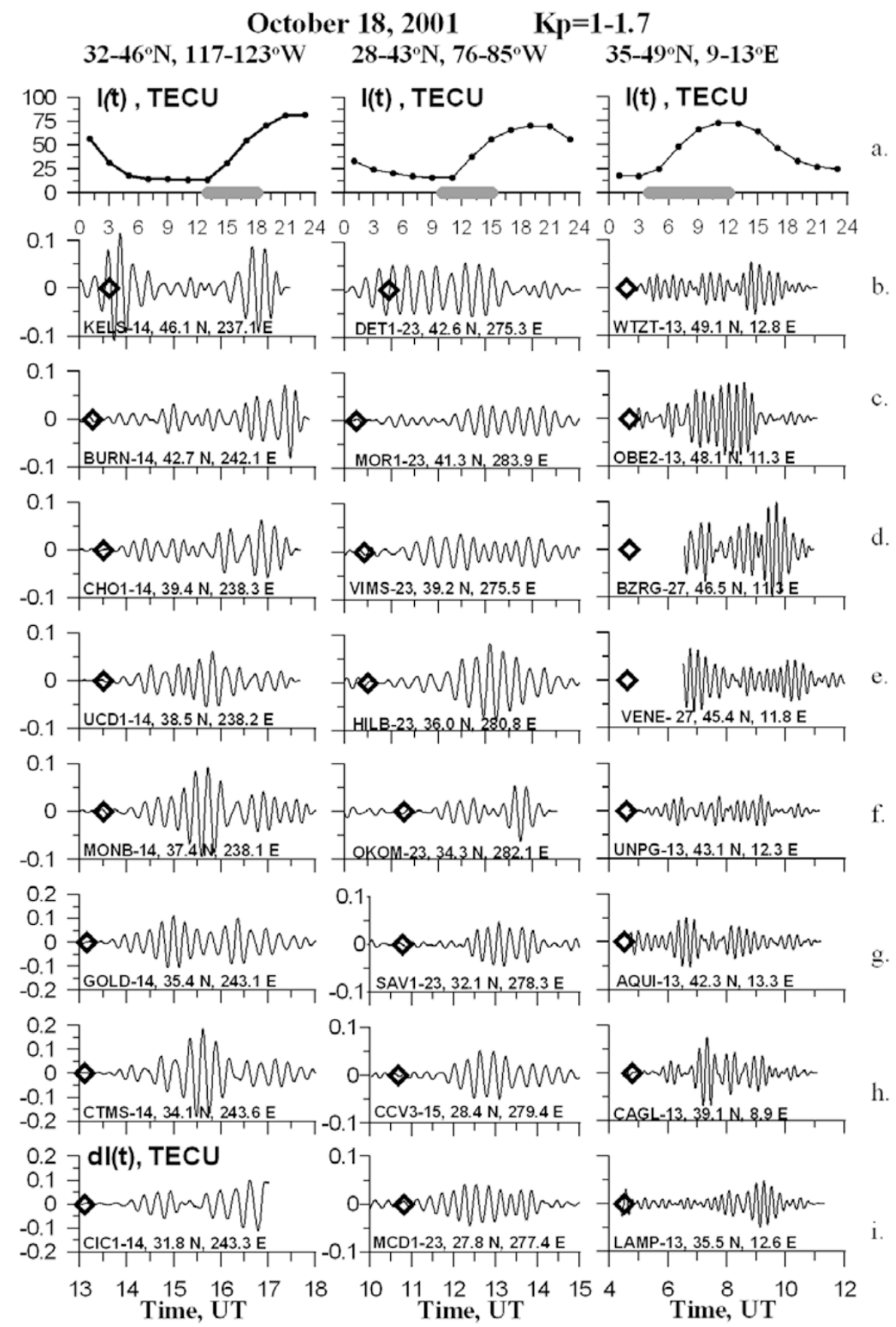

Fig. 3. TEC wave packets for GPS sites located along morning ST over U.S. West-left; over U.S. East-middle; over Europe-right.

of the thermosphere and ionosphere during the sudden sunrise at the dawn side.

The ST-generated WPs (the second type of the observed TEC disturbance) have been found for the first time. The term "wave packets" was first applied to ionospheric disturbances by Hines (1960). Following Hines (1960) and Francis (1974), we decided to use this term for the revealed processes. The investigation reported here has revealed that this designation describes most adequately the phenomenon considered. Particularly, WPs have a limited duration (of about 1-2 h) and follow the ST. Hence, WPs are wave processes for an observer in the fixed coordinate system on the Earth's surface.

These WPs have duration of about $1-2 \mathrm{~h}$ and a time shift of about $1.5-2.5 \mathrm{~h}$ after the ST appearance at $H=100 \mathrm{~km}$. The more-pronounced WP effect can be observed at sunrise; the WP amplitude increases with increasing TEC gradient. The absolute amplitude of TEC WPs is sufficiently small and varies from 0.1 to 0.2 TECU for October 18, 2001 (Fig. 3), and from 0.02 to 0.03 TECU for 3 days over Japan (Fig. 4), which corresponds to the relative amplitude $\frac{d I}{I}$ by about $0.3 \%$ and $0.2 \%$, respectively. Most likely this is the main reason why such a phenomenon has been unknown to date.

The obtained results are in agreement with the theoretical indications of ST effects (Somsikov, 1983) and do not contradict the results obtained by Somsikov (1992) and Drobzhev et al. (1992), which are based on limited statisti- 


\section{GEONET JAPAN}

$$
\text { 249, } 2004(\mathrm{KP}=3.3)
$$

$268,2003(\mathrm{KP}=4.3)$
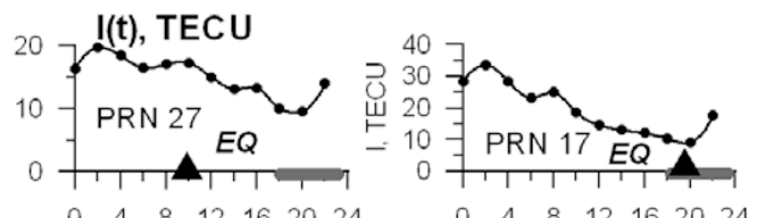

$\begin{array}{lllllllllll}0 & 4 & 8 & 12 & 16 & 20 & 24\end{array}$
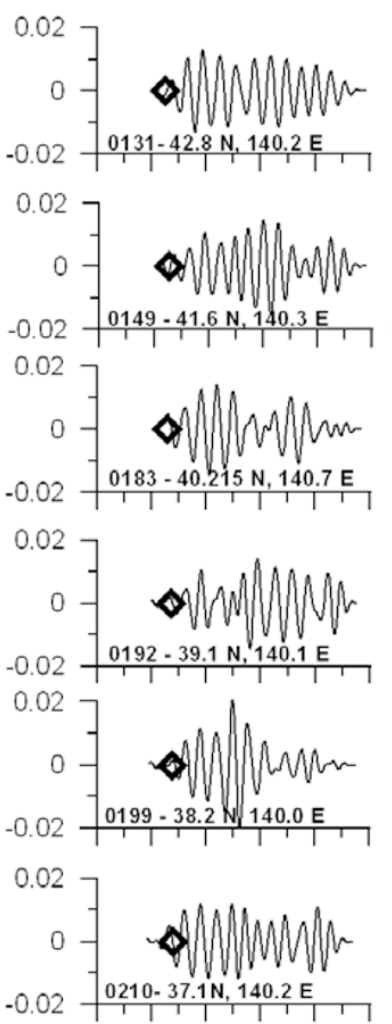

0.02
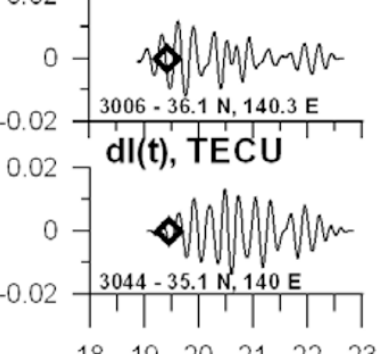

$\begin{array}{llllll}18 & 19 & 20 & 21 & 22 & 23\end{array}$

Time, UT
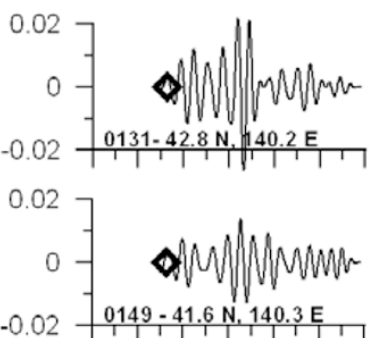

0.02

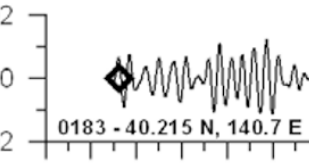

0.03
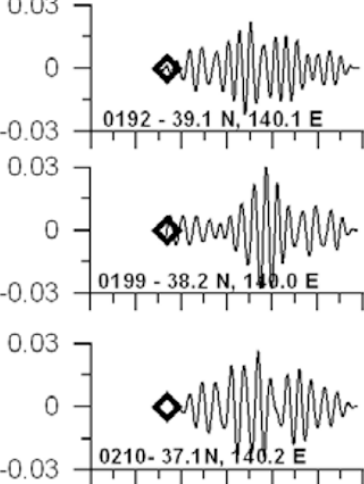

0.03
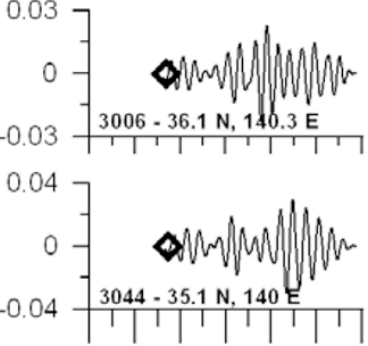

$\begin{array}{lllllll}18 & 19 & 20 & 21 & 22 & 23 & 24\end{array}$

Time, UT
$319,2006(\mathrm{KP}=2.7)$
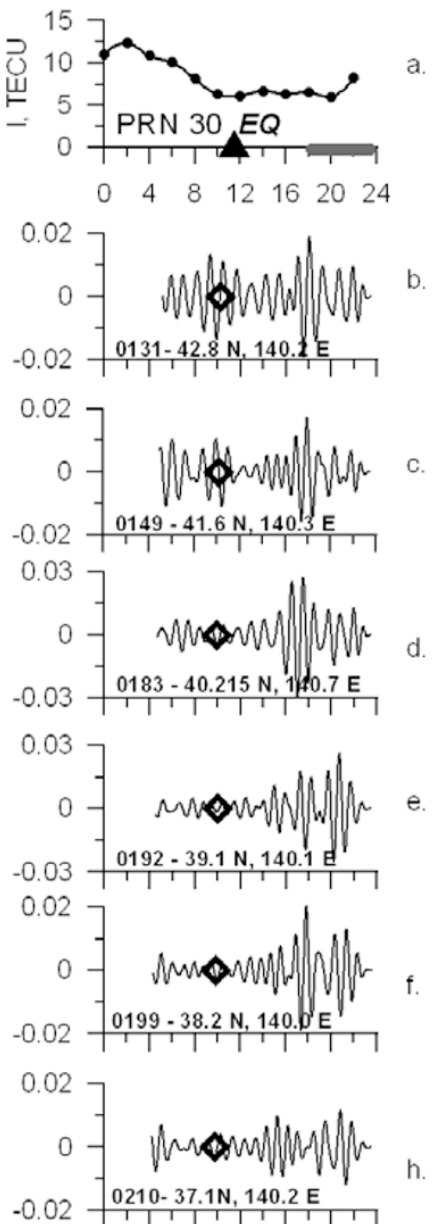

0.03
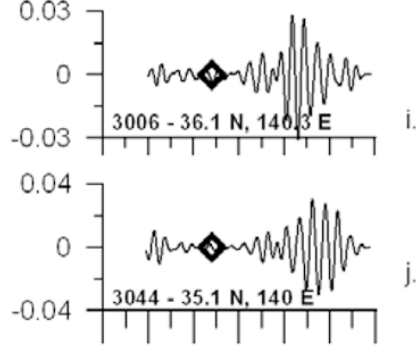

$\begin{array}{lllllll}18 & 19 & 20 & 21 & 22 & 23 & 24\end{array}$

Time, UT

Fig. 4. TEC wave packets for GPS sites located along morning ST over Japan on September 5, 2004-left; on September 25, 2003-middle; on November 15, 2006-right.

cal material. Drobzhev et al. (1992) and Bezotosnyi et al. (1994) studied the dynamic spectra of the radio-wave reflection virtual heights obtained by vertical sounding of the ionosphere. It was shown that during transient hours of the day under magnetically quiet conditions, the low-frequency maximum of the spectra shifts to the higher-frequency region. Our results reinforce this conclusion.

The comparison between our detected WP and the data from Hines (1960) and Francis (1974) suggest that an additional amplitude modulation mechanism exists for wave processes that permits obtaining the closest TEC oscillations in the form of a single WP in the atmosphere. Taking the auroral electrojet as a source of AGW, Francis (1974) showed that the ground-reflected waves gain the properties of a WP, when propagating through the atmosphere into the $F$-region. However, the 2001 October 18 event considered in this paper occurred in the mid-latitude region and at a sufficiently magnetically quiet period, when the conditions of realization of the Francis's mechanism are not satisfied.

The results obtained here do not completely correspond to the known mechanisms of the ionospheric irregularity generation and propagation in various latitudes and can contribute to the development of the theory. Our results are important for developing ionospheric irregularity physics and 
modeling transionosphere radio wave propagation. Spacetemporal features and the nature of the LS TEC and MS TEC structures will be analyzed in detail in future investigations.

Acknowledgments. The author thanks V. A. Medvedev for his interest to the work and fruitful discussion and I. K. Edemskiy, S. V. Voeykov, I. V. Zhivetiev, and Yu. V. Yasukevich for data preparation. We acknowledge the Scripps Orbit and Permanent Array Center (SOPAC), the Crustal Dynamics Data Information System (CDDIS) and GEONET (ftp://terras.gsi.go.jp/data/GPS_products/) for providing GPS data used in this study. The work was supported by the SB RAS and FEB RAS collaboration project N 3.24, the RFBR-GFEN grant N 06-05-39026 and RFBR grant 07-05-00127. Finally, the author wishes to thank the referees for valuable suggestions which greatly improved the presentation of this paper.

\section{References}

Afraimovich, E. L. and N. P. Perevalova, GPS-monitoring of the Earth's upper atmosphere, $480 \mathrm{pp}$, Institute of solar-terrestrial physics, Irkutsk, 2006.

Afraimovich, E. L., N. P. Perevalova, and S. V. Voyeikov, Traveling wave packets of total electron content disturbances as deduced from global GPS network data, J. Atm. Solar-Terr. Phys., 65(11-13), 1245-1262, 2003.

Afraimovich, E. L., S. V. Voeykov, N. P. Perevalova, and K. G. Ratovsky, Large-scale traveling ionospheric disturbances of auroral origin according to the data of the GPS network and ionosondes, Adv. Space Res., doi:10.1016/j.asr.2007.11.023, 2008.

Antonova, V. P., K. E. Dungenbaeva, A. V. Zalizovskii, A. S. Inchin, S. V. Kryukov, V. M. Somsikov, and Yu. M. Yampolskii, Difference between the Spectra of Acoustic Gravity Waves in Daytime and Nighttime Hours due to Nonequilibrium Effects in the Atmosphere, Geomagnetism Aeronomy, 46(1), 101-109, 2006.

Beer, T., On atmospheric wave generation by the terminator, Planet. Space Sci., 26, 185-189, 1978.

Beley, V. S., V. G. Galushko, and Y. M. Yampolski, Traveling ionospheric disturbances diagnostic using HF signal trajectory parameter variations, Radio Sci., 30(6), 1735-1752, 1995.

Bezotosnyi, A. A., V. I. Drobzhev, V. M. Somsikov, A. G. Askarov, and V. A. Belyaev, On peculiarities of variations in the virtual heights of reflection during transient hours of the day, Geomagnetism Aeronomy, 34(3), 401-403, 1994.

Bezrodny, Y. G. and Y. M. Yampolski, On the origin of the spatial phase deference of the VLF at sunrise and sunset, Radiophys. Quantum Electronics, 19(9), 1270-1274, 1976.

Cot, C. and H. Teitelbaum, Generated of the gravity waves by inhomogeneous heating of the atmosphere, J. Atmos. Terr. Phys., 42(9/10), 877$883,1980$.

Drobzhev, V. I., D. E. Zachateisky, P. E. Kozina et al., Mid-latitude shortperiod disturbances in the ionosphere during the solar terminator passage, Geomagnetism Aeronomy, 32(2), 181-183, 1992.
Dungenbaeva, K. E. and B. Ganguly, Radiation changes in atmospheric wave dynamics and spectra, Math. Comput. Simulation, 67, 411-417, 2004.

Francis, S. H., A theory of medium-scale traveling ionospheric disturbances, J. Geophys. Res., 79, 5245-5259, 1974.

Galushko, V. G. and Y. M. Yampolski, Experimental investigations of the HF signals scattered by moving solar terminator, Radiophys. Quantum Electronics, 26(4), 499-502, 1983.

Galushko, G., V. V. Paznukhov, Y. M. Yampolski, and J. C. Foster, Incoherent scatter radar observations of AGW/TID events generated by the moving solar terminator, Ann. Geophys., 16, 821-827, 1998.

Galushko, V. G., V. S. Beley, A. V. Koloskov, Y. M. Yampolski, B. Reinisch, and V. V. Paznukhov, Frequency-and-Angular HF Sounding and VHF ISR Diagnostics of TIDs, Radio Sci., 38(6), 1102-1110, 2003.

Hines, C. O., Internal atmospheric gravity waves at ionospheric heights, Canadian J. Phys., 38(8), 1441-1481, 1960.

Hocke, K. and K. Schlegel, A review of atmospheric gravity waves and traveling ionospheric disturbances: 1982-1995, Ann. Geophys., 14, 917-940, 1996.

Hocke, K. and K. Igarashi, Electron density in the F region derived from GPS/MET radio occultation data and comparison with IRI, Earth Planets Space, 54, 947-954, 2002.

Hofmann-Wellenhof, B., H. Lichtenegger, and J. Collins, Global Positioning System: theory and practice, 327 pp, Springer-Verlag Wien., New York, 1992.

Klobuchar, J. A., Ionospheric time-delay algorithm for single-frequency GPS users, IEEE Trans. Aerospace Electronics System, 23(3), 325-331, 1986.

Mishin, E. V., A. E. Epishova, L. M. Ishkova, E. M. Kovalevskaia, E. F. Koz lov, L. E. Kolokolov, L. N. Rubcov, N. I. Samorokin, L. N. Sidorova, V. M. Somsikov, V. A. Telegin, and L. A. Ydovich, Disturbances of F-region electron density following solar terminator during the WITS period of 16-20 March, J. Atmos. Terr. Phys., 33(4/5), 643648, 1991.

Popov, A. V. and Y. M. Yampolski, VLF variations during sunrise period, Radiophys. Quantum Electronics, 24(6), 794-798, 1981.

Sobral, J. H. A., G. L. Borba, M. A. Abdu, I. S. Batista, H. Sawant, C. J. Zamlutti, H. Takahashi, and Y. Nakamura, Post-sunset wintertime 630.0 $\mathrm{nm}$ airglow perturbations associated with gravity waves at low latitudes in the South American sector, J. Atmos. Terr. Phys., 59, 1611-1623, 1997.

Somsikov, V. M., Solar terminator and dynamics of the atmosphere, 192 pp, Nauka, Alma-Ata, 1983.

Somsikov, V. M., A spherical model of wave generation in atmosphere by solar terminator, J. Atmos. Terr. Phys., 49(5), 433-438, 1987.

Somsikov, V. M., On generation of the atmosphere turbulence by the solar terminator, Geomagnetism Aeronomy, 32(3), 55-59, 1992.

Somsikov, V. M. and B. Ganguly, On the mechanism of formation of atmospheric inhomogeneties in the solar terminator region, J. Atmos. Terr. Phys., 57, 75-83, 1995.

E. L. Afraimovich (e-mail: afra@iszf.irk.ru) 